\title{
3-D Localization Schemes of RFID Tags with Static and Mobile Readers
}

\author{
Mathieu Bouet and Guy Pujolle \\ Université Pierre et Marie Curie (UPMC) - Laboratoire d'Informatique de Paris 6 (LIP6) \\ 104 avenue du Président Kennedy, \\ 75016 Paris, France \\ \{Mathieu.Bouet, Guy.Pujolle\}alip6.fr
}

\begin{abstract}
RFID is an automatic identification technology that enables tracking of people and objects. We propose a scalable and robust 3-D localization method of RFID tags, derived in two schemes, that uses the diversity of pervasive environments to improve its accuracy. RFID readers are placed on the floor and ceiling of a room. In the first scheme, static and mobile tags are localized using only connectivity information obtained through this structure. The second scheme involves in addition mobile RFID readers. They are used here to refine the localization process by adding more inclusive constraints to the calculation. We study the impact of the number of static and mobile readers on the accuracy. We then show that the first scheme constitutes an upper bound for the second one. Furthermore, we observe that the second scheme does not require a lot of mobile readers and that it considerably improves accuracy.
\end{abstract}

Keywords: RFID, localization, mobility, 3 dimensions, tracking.

\section{Introduction}

Radio Frenquency IDentification (RFID) has recently become widespread for automatic identification and tracking. A RFID system is composed of two types of entities: RFID tags (or transponders) that store identity, and RFID readers (or detectors) that remotely retrieve these data. Tags can be passive: communications are supplied by the reader generally by backscattering its carrier wave, semi-passive: the tags embed a power source for their internal processes and communicate with readers like passive tags, and active: they embed a battery that supplies communications with readers and functionalities such as cryptography or sensor. Active tags are more expensive than passive tags but their communication range is far larger and they can have more advanced utilizations [1]

RFID is a key technology in future pervasive systems and services. Indeed, it provides essential context-aware functionalities such as automatic identification, tracking, and real-time inventory. By building RFID in networks, new management concepts could be conceived so as to fully integrate real-time status of items and people. However, all the benefits of this technology could be hugely increased if identification information was linked to positions. It would add another dimension to the context-awareness: real-time location and consequently mobility. For example, it could be used in home-networking and libraries to retrieve specific objects, control 
access or monitor events. Matching identities with their locations would also allow the development of new strategies in autonomic systems for mobility control, resource allocation, security, service discovery. Classic RFID systems only provide coarse-grained localization. In fact, they are generally composed of readers placed in strategic locations; their purpose is to identify all the tags that pass in their read range. As a matter of fact, the precision of the position corresponds to the size of the cell formed by their read range.

In this paper, our major contribution is to design a 3-D localization algorithm for mobile and static RFID tags which, contrary to other related works, is based only on connectivity information and which takes into account mobile RFID readers. Our algorithm offers fine-grained position estimation and is conceived so as to be cheap, robust, fast, and thus scalable. Moreover mobile readers are used to refine and improve the precision. Our model is simple: we consider a room, a warehouse, or a container (modeled by a hexahedron) and we deploy readers on the floor and ceiling. No assumption is done on the equipments and their quantity is reduced to the minimum in order to have a low-cost system. In the first scheme, an estimated position is computed for each mobile or static tag by using only connectivity information (which reader can detect the tag and which cannot) with a semi-infinitely constrained multivariable nonlinear method. Then, we present a second method, derived from the first one, to increase the localization precision when there are mobile readers. They are used to refine the first method by adding more constraints. This calculation requires more time and can only be applied on static RFID tags. First, we evaluate the impact of the number of readers on the precision of the localization, and we show that the accuracy of our method is very high when they are densely deployed. Then, we study the impact of the number of mobile readers on the performance of the second method, we underline the existence of an upper bound for the precision, and we point out a quick and impressive reduction of the localization error.

The rest of the paper is organized as follows. First we discuss related work in Section 2. Then we explain the basic localization method and the improved localization method with mobile readers in Section 3. Simulation methodology and results are discussed in Section 4. Finally, Section 5 concludes the paper.

\section{Related Work}

Localization is often considered as a widely studied domain. However, few methods have been proposed for RFIDs. In fact, this area has specific constraints and requirements. Contrary to localization in Mobile Ad-hoc NETworks (MANETs) or in Wireless Sensor Networks (WSNs) for which a lot of algorithms have been conceived, a RFID system is centralized because the computation capabilities of RFID tags are very limited. Besides they are composed of tens of thousands of tags that need to be treated quasi simultaneously. Moreover, they are usually deployed in indoor environments and their signal is harshly impaired. For example, UHF tags' radio waves do not bounce off metal and have difficulties to penetrate water.

The most famous indoor localization sensing method is called LANDMARC (LocAtioN iDentification based on dynaMic Active Rfid Calibration) [2]. It only 
localizes active RFID. The system is calibrated with the help of reference tags (additional tags that are fixed and whose positions are known by the system): a map including the Received Signal Strength (RSS) of each reader is built. Then, the system has to retrieve the RSS information from each tag to the readers and checks the map to find out its estimated position. Another well-known RFID localization system is SpotON [3]. It is also based on RSS measurements and uses them to estimate the distance between readers and tags. A central server aggregates the data and performs a trilateration to obtain the position of the tags. In [4], the authors also estimate the distance between tags and readers. However, this estimation is done through the Time Difference Of Arrival (TDOA) technique: the system measures the time-of-flight and deduces the distance according to the frequency and the inter-delay. This scheme is designed for fixed passive SAW RFID tags. Finally, in [5] the authors propose a 3-D localization scheme. It is founded on the assumption that readers have numerous power levels and on the deployment of reference tags. The read range of each reader is increased or decreased until the tag is detected. Then, thanks to the reference tags, an upper and a lower bound are obtained. The localization is done by mixing the bounds of all the readers and by applying a time-costly computation on the obtained area.

Distance estimation through RSS, TOA or AOA is often very complicated in indoor environments because of signal absorption, indirect path, and interferences. These techniques can lead to large errors and require specific and thus expensive equipments. Moreover, the use of reference tags considerably increases the whole cost of the system and necessitates manual installation. Our localization method is based on very simple principles in order to be cost-effective, robust in harsh environments, and fast enough to be scalable when thousands of items need to be treated simultaneously. Indeed, we consider readers with basic capabilities, we do not use any additional equipment, and we localize tags using only connectivity information, no distance estimation is performed.

General indoor localization systems have also been studied for a long time. The three more famous are Cricket [6], Active Bat [7], and Active Badge [8]. In the last system, Active Badges transmit unique infra-red signals every 10 seconds. When they pass close to a reader, they are detected. The position of the badge corresponds to the one of the reader that detected it, and the precision is the size of the cell. In Active Bat, ultrasonic receivers (or sensors) are placed on the ceiling. The system measures the time-of-flight from the node to each receiver and applies a classic trilateration. Finally, Cricket is also based on TDOA measurements but the difference is that the sensors send their coordinates to the node and it is the node that performs the trilateration to estimate its position.

Several location methods for geographic routing and security mechanisms have also been proposed for MANETs and WSNs. The vast majority of them measures inter-node distance through the RSS, TDOA, or Angle of Arrival (AOA) technique. The others are based on hop count or inclusive and exclusive constraints. The readers are referred to [9] for more details. As explained earlier, those approaches address different problems than the one treated in this article but they offer a good comprehension and a solid basis for our approach. This also goes for the numerous methods that use reference RFID tags to localize mobile robots. 


\section{Our Algorithm}

\subsection{System Setup}

First of all, we place RFID readers on the floor and ceiling of a room, a warehouse, or a container. These are modelized by a hexahedron whose length, width, and height are $\mathrm{L}, \mathrm{W}$, and $\mathrm{H}$ respectively as shown in Fig. 1. The readers are spaced from each other according to a certain gap $\mathrm{S}$. The exact position of each reader is known by the system. Furthermore, they are supposed to be as basic as possible, that is, they have no different power levels, no directional antenna, no multiple antennas, and no synchronization. They can also have different read ranges. For easier comprehension, we consider they all have the same capacities and thus the same read range $\mathrm{R}$, and we assume the signal transmission between readers and tags forms a sphere. Both active and passive tags can be located with this system.

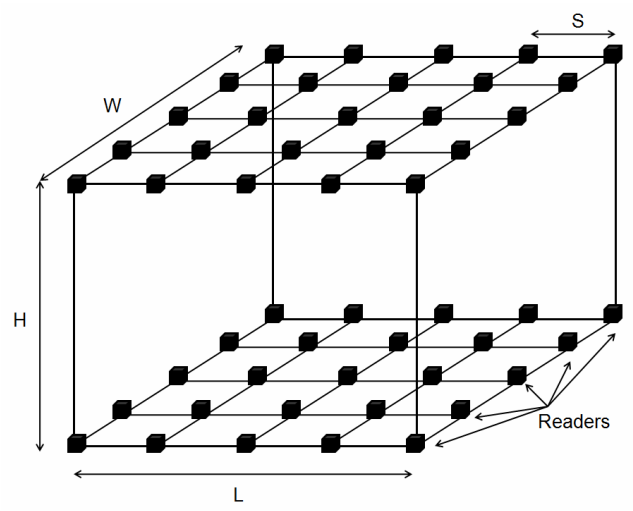

Fig. 1. Our model

\subsection{Basic Position Estimation}

This first method can locate both static and mobile tags and is based on the architecture presented in Section 3.1. The localization of tags only requires connectivity information: which readers are able to detect a given tag and which cannot. No distance estimation is performed. A mean position is calculated for each combination of intersection of readers as shown in Fig. 2. These mean positions correspond to the barycenter (also known as the center of gravity or the center of mass) of the different volumes formed by the intersection of the readers' detection areas.

Tags' positions are estimated as follows. First, we define an error objective function $\delta_{i}(x, y, z)$ for each combination of readers:

$$
\delta_{i}(x, y, z)=\sum_{j=1}^{n_{j}} \sqrt{\left(x-x_{j}\right)^{2}+\left(y-y_{j}\right)^{2}+\left(z-z_{j}\right)^{2}} .
$$




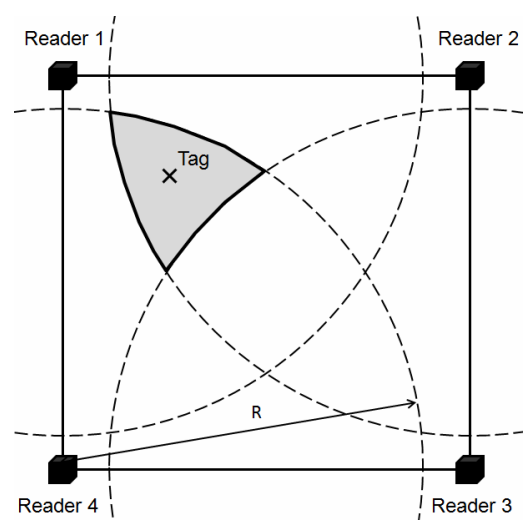

Fig. 2. A 2 dimensional intersection of readers

Where $n_{j}$ is the number of readers that form the combination, $(x, y, z)$ are the unknown coordinates of the barycenter (that will be the estimated position), and $\left(x_{j}, y_{j}, z_{j}\right)$ the coordinates of the reader $j$.

The error objective function is defined as the sum of the distance between the tag whose position is unknown and every reader able to read it.

Then, as the number of readers on the floor is equal to the one on the ceiling, we can easily deduce if the tag is close to the floor, in the middle zone, or close to the ceiling. Indeed, if the tag is near the floor, more readers on the floor than on the ceiling will be able to read it. In that case, we can add extra information to the error objective function in equation (2). The same is done if more readers on the ceiling than on the floor are activated in equation (3). Nothing is done if the quantities are equal.

$$
\begin{aligned}
& \delta_{i}(x, y, z)=\delta_{i}(x, y, z)+\sqrt{\left(z-z_{\text {floor }}\right)^{2}} \\
& \delta_{i}(x, y, z)=\delta_{i}(x, y, z)+\sqrt{\left(z-z_{\text {ceiling }}\right)^{2}} .
\end{aligned}
$$

Finally, we use a semi-infinitely constrained multivariable nonlinear method to find the minimum of the error objective function presented in equation (1). It takes as parameter non linear functions, linear inequalities (the difference between the maximum read range $\mathrm{R}$ and the distance reader-unknown estimated position), and lower and upper bounds (the dimensions of the hexahedron). This well-known algorithm uses cubic and quadratic interpolation techniques to estimate peak values in the semi-infinite constraints. The error objective function has been defined so that its minimum corresponds to the barycenter of the volume: the calculated point will minimize the sum of the distances with all the readers plus the distance with the floor or the ceiling. The problem is stated as follows: 


$$
\begin{aligned}
& \min _{(x, y, z)} \delta_{i}(x, y, z) \text { subject to } \\
& \left\{\begin{array}{l}
0 \leq x \leq L \\
0 \leq y \leq W \\
0 \leq z \leq H \\
\sqrt{\left(x-x_{1}\right)^{2}+\left(y-y_{1}\right)^{2}+\left(z-z_{1}\right)^{2}}-R \leq 0 \\
\cdots \\
\sqrt{\left(x-x_{n_{j}}\right)^{2}+\left(y-y_{n_{j}}\right)^{2}+\left(z-z_{n_{j}}\right)^{2}}-R \leq 0
\end{array}\right.
\end{aligned}
$$

Where $\left(x_{1}, y_{1}, z_{1}\right), \ldots,\left(x_{n_{j}}, y_{n_{j}}, z_{n_{j}}\right)$ are the coordinates of the readers that formed the volume.

It is obvious that if the result for a given combination has already been computed, it is not necessary to recompute it. Consequently, this localization method can require a few calculations at the beginning but will be very fast when all the cases are treated. Actually, a solution would be to calibrate the room at the start, i.e. compute the corresponding mean position of every combination of reader. The localization would then be very fast: check the built map and retrieve the tag's position. This process can be easily applied both on static and mobile tags since only connectivity information is used.

\subsection{Position Estimation Enhanced by Mobile Readers}

In realistic pervasive environments, RFID readers can be mobile. We propose to use this diversity to improve our localization scheme.

We lean this method on the first one: the room is equipped in the same way and the positions are estimated through the same process if no mobile reader is present. The mobile readers do not need to be complex or expensive since, once again, we deduce connectivity information from their location and their maximum read range. Their positions are known by the system. The interaction between the static reader structure and the mobile readers is shown in Fig. 3.

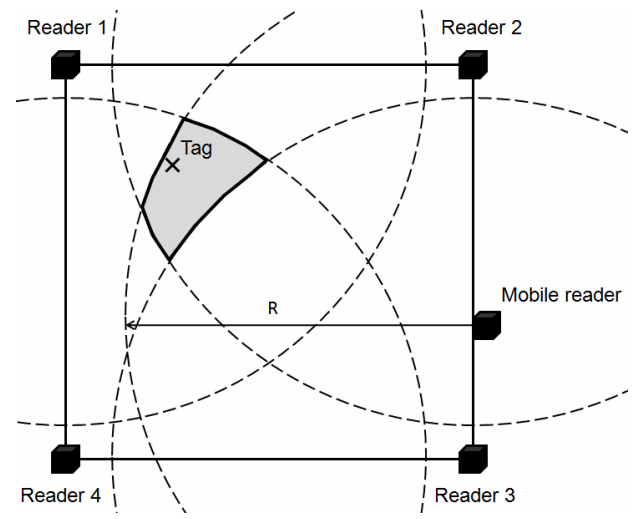

Fig. 3. A 2 dimensional intersection of static and mobile readers 
Consequently, the equation (4) becomes:

$$
\begin{aligned}
& \min _{(x, y, z)} \delta_{i}(x, y, z) \quad \text { subject to } \\
& \left\{\begin{array}{l}
0 \leq x \leq L \\
0 \leq y \leq W \\
0 \leq z \leq H \\
\sqrt{\left(x-x_{1}\right)^{2}+\left(y-y_{1}\right)^{2}+\left(z-z_{1}\right)^{2}}-R \leq 0 \\
\cdots \\
\sqrt{\left(x-x_{n_{j}}\right)^{2}+\left(y-y_{n_{j}}\right)^{2}+\left(z-z_{n_{j}}\right)^{2}}-R \leq 0 \\
\sqrt{\left(x-x_{\text {mobile }_{k}}\right)^{2}+\left(y-y_{\text {mobile }_{k}}\right)^{2}+\left(z-z_{\text {mobile }_{k}}\right)^{2}}-R \leq 0
\end{array}\right.
\end{aligned}
$$

Where $\left(x_{\text {mobile }_{k}}, y_{\text {mobile }_{k}}, z_{\text {mobile }_{k}}\right)$ are the coordinates of the mobile reader that detected the tag.

Each time a mobile reader performs the reading of a tag, an inclusive constraint is added. Obviously, if the tag is static, this scheme can be easily applied and the zone is reduced quickly or not depending on the number of mobile readers. If the tag is moving slowly, it can also be localized with this variant but it is necessary to put timestamp on the constraints defined by the mobile readers and to remove the oldest ones. If the tag is moving quickly, the first localization method has to be applied.

\section{Simulation}

\subsection{Simulation Methodology and Settings}

We have run extensive simulations in order to study the accuracy of our methods. The semi-infinitely constrained multivariable nonlinear algorithm has been implemented in several mathematic programs. We chose the one in the Optimization Toolbox available in Matlab [10].

We have defined different error measurements so as to evaluate the accuracy:

- The mean error in 2 dimensions:

$$
\mathcal{E}_{2 D}=\sqrt{\left(x_{\text {real }}-x_{\text {estimated }}\right)^{2}+\left(y_{\text {real }}-y_{\text {estimated }}\right)^{2}},
$$

And its standard deviation $\sigma_{2 D}$.

- The mean error in 3 dimensions:

$$
\varepsilon_{3 D}=\sqrt{\left(x_{\text {real }}-x_{\text {estimated }}\right)^{2}+\left(y_{\text {real }}-y_{\text {estimated }}\right)^{2}+\left(z_{\text {real }}-z_{\text {estimated }}\right)^{2}},
$$

And its standard deviation $\sigma_{3 D}$. 
- The mean error regarding the estimated height:

$$
\varepsilon_{h}=\sqrt{\left(z_{\text {real }}-z_{\text {estimated }}\right)^{2}},
$$

And its standard deviation $\sigma_{h}$.

We dimensioned the hexahedron as follows:

$$
\begin{aligned}
& L=100 m \\
& W=100 m \\
& H=3 m
\end{aligned} .
$$

Where $\mathrm{L}, \mathrm{W}$, and $\mathrm{H}$ are the length, the width, and the height respectively. These values have voluntarily been chosen bigger than the dimensions of a typical room so as to take into account all the possible shapes.

We choose realistic values for the read range of the static and mobile RFID readers:

$$
R=5 m .
$$

Finally, we implemented the Random WayPoint Mobility Model (RWP) [11] for the mobile readers. This model is used in many works since it is relevant for simulation of people displacements. The mobile readers randomly pick a destination in the hexahedron, go to it with a constant speed, when they reach it they pause, and then start the same process. It would be better if the mobile readers were not all at the same height but for an easier analysis of the performances, we chose to place them at 1 meter from the floor and to make them move with the speed of someone who is walking (5 kilometers/hour).

Since the related works presented in Section 2 do not provide enough accurate hypotheses or use different configurations and different equipments (such as reference tags) from our model, we are not able to propose any comparison.

\subsection{Results}

Fig. 4 shows the mean error in two dimensions (on $x$ and $y$ coordinates) after 300 seconds for different quantities of mobile readers. The accuracy of our basic localization scheme is below 1 meter when the static readers are spaced until 6 meters. A local peak is observed at 4.5 meters. This is due to the shape of the intersections of readers' areas. Indeed, this value is just below the one of the radius. The readers on the floor and ceiling thus form very irregular zones, hence a higher error. This data constitutes an upper bound for the second localization method. After 300 seconds, with only one mobile reader the 2-D error is reduced until 40 centimeters compared to the static results. In the same time, if 5 mobile readers are used, the estimated error falls between 18 and 57 centimeters. Furthermore, the use of 10 or 20 mobile readers almost cancels the impact of the inter-reader spacing since after 300 seconds the error is bounded between 17 and 23 centimeters. 


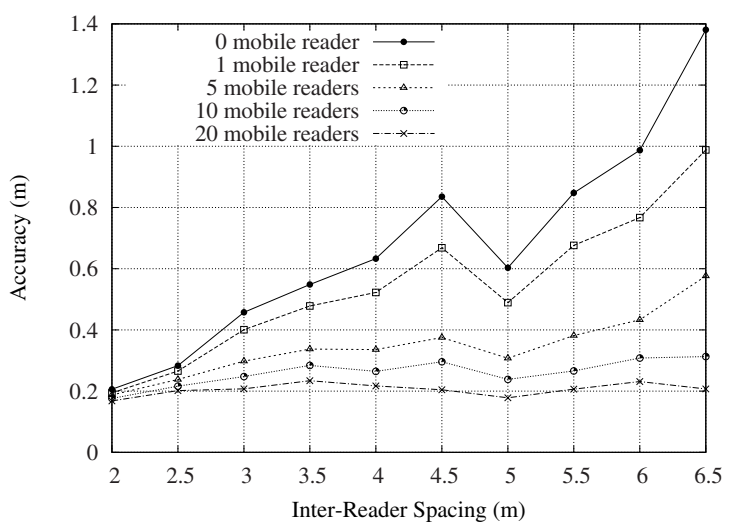

Fig. 4. 2-D accuracy after 300 seconds

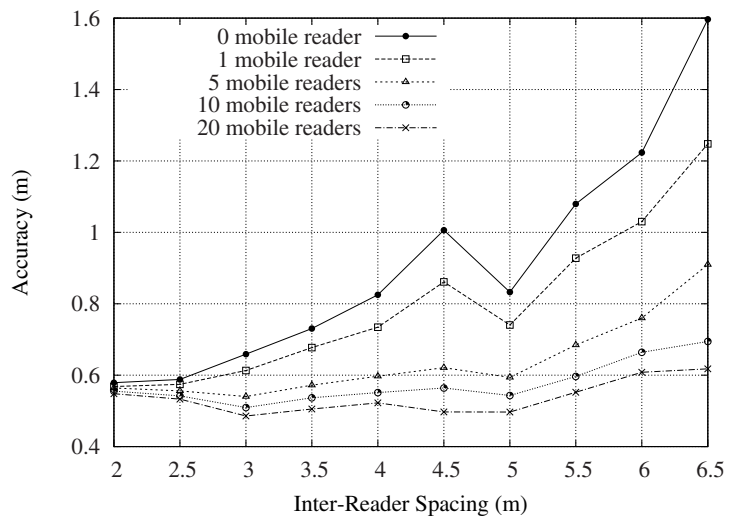

Fig. 5. 3-D accuracy after 300 seconds

Fig. 6 shows the mean error on the estimated height. It is limited between 39 and 61 centimeters. When the readers are densely deployed, the shapes of the intersections formed by the readers' areas are not as regular as the ones when the inter-spacing reader is around 3 or 3.5 meters. The same phenomenon occurs in the cases where they are far one another. Consequently, the accuracy is better around $3-3.5$ meters. The results for the second localization scheme are roughly the same. However, we can notice an improvement of the accuracy when mobile readers are used with static readers spaced of at least 4 meters. The impact of the mobile readers could be hugely increased if they were not all placed at the same height and if their read range were not all equal. For easier comparison between the different scenarios we chose identical mobile readers.

Fig. 5 shows the 3-D mean error. The curbs have almost the same shapes than the ones for the 2-D results. This can be explained by the fact that our second localization method has only a light impact on the improvement of the height accuracy. Therefore, 


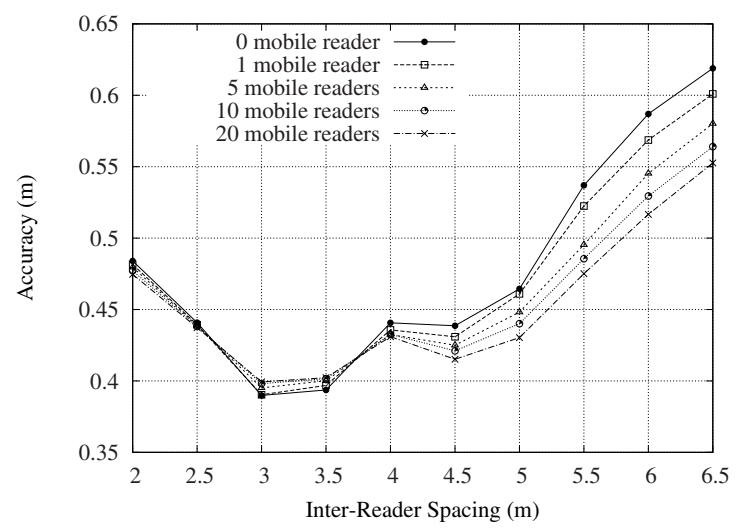

Fig. 6. Accuracy on height after 300 seconds

the evolution of the 3-D accuracy tends to follow the one in 2-D. The error is bounded between 57 and 159 centimeters for the first localization method. When 20 mobile readers are used, after 300 seconds the error falls between 48 and 61 centimeters (until $62 \%$ of reduction).

The evolution of the accuracy through time is illustrated in Fig. 7. The impact of the mobile readers is visible since the beginning. After 30 seconds, the precision has been greatly increased and it goes on over time. Nevertheless, we can notice that improvement of the accuracy tends to decrease since the difference between the results at 240 seconds and at 300 seconds is only a few centimeters.

Finally, in Fig. 8 we compare the impact of the number of mobile readers on the accuracy. Since for the first localization scheme there is no mobile reader, the error remains unchanged through time. In addition, two interesting remarks can be done. First, it is not necessary to use a lot of mobile readers. Indeed, the difference between

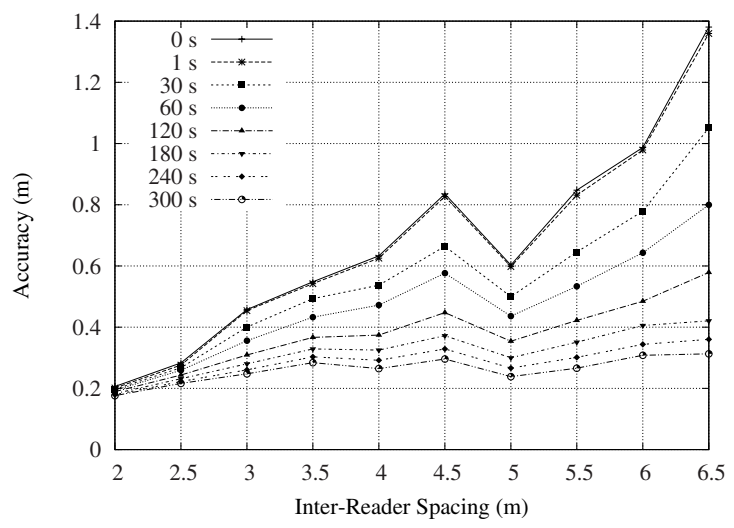

Fig. 7. Evolution of the 2-D accuracy with 10 mobile readers 


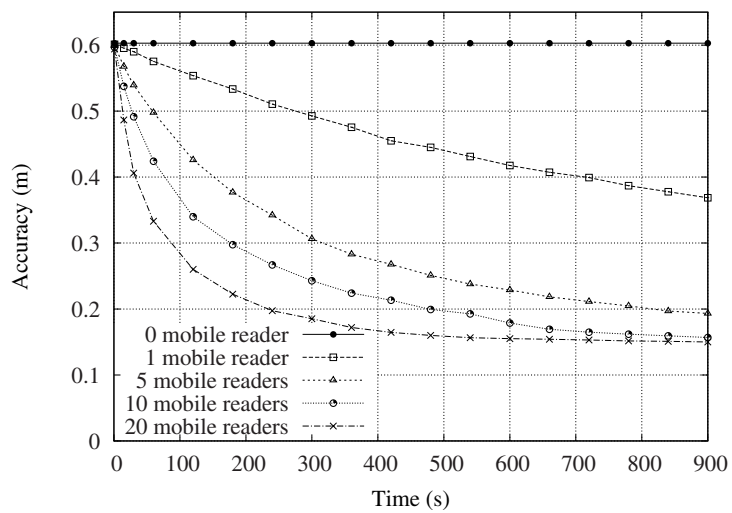

Fig. 8. Evolution of the 2-D accuracy for an inter-spacing of 5 meters

the results for 5 mobile readers and the ones for 10 and 20 is not as important as expected. Secondly, as noticed in Fig. 7, the mean error in each scenario tends to rapidly converge over time. A residual error remains because of the inter-spacing between the static readers.

To sum up, the results for the first localization scheme constitute an upper bound for the mean error of the second scheme. The use of mobile readers improves the accuracy. It depends on the number of mobile readers, the space between the static readers, and the execution time. For example, when inter-space is 5.5 meters and when 10 mobile readers are involved, the 3-D error falls from 92 to 59 centimeters (a reduction of 36\%) after 300 seconds. Moreover, the results show that the error reduction converges quickly over time and that it does not require a lot of mobile readers.

\section{Conclusion}

We have proposed a 3 dimensional localization method for static and mobile RFID tags and we have shown how to use diversity of pervasive environments, that is to say mobile RFID readers, to improve the positioning accuracy. We have chosen very simple properties so as to obtain a robust, fast, cost-efficient, and thus scalable method. Static RFID readers are placed on the floor and ceiling of a room. Tags' positions are estimated by using only connectivity information in a semi-infinitely constrained multivariable nonlinear method. We then derive this scheme: mobile readers are used to refine the inclusive constraints and thus to improve the accuracy of static tags' estimated location. Our results show that the precision of the first scheme is very satisfactory compared to the requirements. Furthermore, the use of mobile readers hugely reduces the localization error. The analysis also shows that it is not necessary to involve a lot of mobile readers and that the error reduction rapidly converges making the second localization scheme not only very quick but also very precise. 


\section{References}

1. Want, R.: An introduction to RFID technology. IEEE Pervasive Computing 5(1), 25-33 (2006)

2. Ni, L.M., Liu, Y., Lau, Y.C., Patil, A.P.: LANDMARC: indoor location sensing using active RFID. In: First IEEE International Conference on Pervasive Computing and Communications, pp. 407-415 (2003)

3. Hightower, J., Borriello, G., Want, R.: SpotON: An Indoor 3D Location Sensing Technology Based on RF Signal Strength. UW CSE Technical Report (2000)

4. Bechteler, T.F., Yenigun, H.: 2-D localization and identification based on SAW ID-tags at 2.5 GHz. IEEE Transactions on Microwave Theory and Techniques 51(5), 1584-1590 (2003)

5. Wang, C., Wu, H., Tzeng, N.-F.: RFID-Based 3-D Positioning Schemes. In: 26th IEEE International Conference on Computer Communications (INFOCOM), pp. 1235-1243 (2007)

6. Priyantha, N.B., Chakraborty, A., Balakrishnan, H.: The cricket location-support system. In: 6th Annual ACM International Conference on Mobile Computing and Networking (MOBICOM), pp. 32-43 (2000)

7. Ward, A., Jones, A., Hopper, A.: A New Location Technique for the Active Office. IEEE Personal Communications 4(5), 42-47 (1997)

8. Want, R., Hopper, A., Falcao, V., Gibbons, J.: The active badge location system. ACM Transactions on Information Systems 10(1), 91-102 (1992)

9. Gustafsson, F., Gunnarsson, F.: Mobile positioning using wireless networks: possibilities and fundamental limitations based on available wireless network measurements. IEEE Signal Processing Magazine 22(4), 41-53 (2005)

10. Matlab, http://www.mathworks.com/

11. Hyytia, E., Lassila, P., Virtamo, J.: Spatial node distribution of the random waypoint mobility model with applications. IEEE Transactions on Mobile Computing 5(6), 680-694 (2006) 